\title{
An Approximate Dynamic Programming Algorithm for the Allocation of High-Voltage Transformer Spares in the Electric Grid
}

\author{
Johannes Enders \\ Department of Operations Research and Financial Engineering \\ Princeton University \\ Warren B. Powell \\ Department of Operations Research and Financial Engineering \\ Princeton University \\ David Egan \\ PJM Interconnection
}

May 25, 2009 


\begin{abstract}
This paper addresses the problem of allocating high-voltage transformer spares throughout the electric grid to mitigate the risk of random transformer failures. With this application we investigate the use of approximate dynamic programming (ADP) for solving large scale stochastic facility location problems. The ADP algorithms that we develop consistently obtain near optimal solutions for problems where the optimum is computable and outperform a standard heuristic on more complex problems. Our computational results show that the ADP methodology can be applied to large scale problems that cannot be solved with exact algorithms.
\end{abstract}




\section{Introduction}

High-voltage transformers are an integral part of the electric transmission system. A catastrophic transformer failure constitutes the most severe failure event and usually requires the replacement of the transformer. Catastrophic failures can be extremely costly if they require more expensive generation to be brought online in order to relieve system congestion. We refer to the additional costs that are due to a transformer failure as congestion costs. As many of the high-voltage transformers in the U.S. have been installed in the 1960s and 70s, transmission owners and operators have become increasingly concerned with the growing failure risk of these older transformers.

We study the system of $500 \mathrm{kV}$ to $230 \mathrm{kV}$ transformers that are operated by PJM Interconnection (PJM). PJM operates the electric grid in all or parts of Delaware, Illinois, Indiana, Kentucky, Maryland, Michigan, New Jersey, North Carolina, Ohio, Pennsylvania, Tennessee, Virginia, West Virginia, and the District of Columbia. This service area has a population of about 51 million. The approximately $200500 / 230 \mathrm{kV}$ transformers in this area are owned by 11 different transmission owners (TOs).

We address the problem of planning spare transformers to respond to random failures. Spares are crucial because the lead time to obtain a new transformer to replace a failed one can be 12 to 18 months or longer depending on the order books of transformer manufacturers. For high-voltage transformers the issue of where to locate transformer spares in the transmission network is of particular importance. These transformers weigh up to 200 metric tons and as a result the cost and time involved in moving them are significant. Their transportation needs special permits, and may require the reinforcement of bridges and the restoration of rail access

to a transformer substation. Considerable congestion costs may be incurred during the time it takes to transfer a spare to a failure site. Hence, moving spares quickly is an important task. In this paper we consider the problem of placing a given number of spares in the network such that the expected costs associated with random transformer failures are minimized. By running our model repeatedly for different numbers of spares we can also address the issue of spare quantity which is of considerable practical importance. 
The problem of planning spare transformers can be formulated as a multistage stochastic, dynamic program of a very large size. A growing body of research in approximate dynamic programming has demonstrated that these techniques scale to very large problems, with a virtually unlimited ability to handle complex operational details (see, for example, Bertsekas \& Tsitsiklis (1996), Powell et al. (2005), Topaloglu \& Powell (2006)). The basic strategy in ADP is to simulate forward in time, iteratively updating estimates of value functions that approximate the value of being in a state. As with many stochastic optimization algorithms, multistage problems are reduced to sequences of two-stage problems. In our setting, this two-stage problem consists of allocating transformers to different locations, followed by a random realization of failures around the network. If our technique is going to be successful for multistage problems, it has to be able to provide good solutions to the two-stage problem. In this paper, we consider only the two-stage problem, but we use techniques that generalize easily to multistage problems, where we will be interested in 50 year horizons.

The two-stage allocation problem has the behavior of a two-stage stochastic facility location problem where we allocate the spares to locations, then observe random failures after which we have to move the spares to the locations where the failures occurred. The framework of twostage stochastic programming is presented in Birge \& Louveaux (1997) and Kall \& Wallace (1994). The allocation problem we present here is integer in nature and thus the stochastic integer programming literature is relevant. Sen (2005) provides a comprehensive overview of the state of the art in stochastic integer programming. Stochastic facility location problems (Louveaux \& Peeters (1992), Laporte et al. (1994), Ntaimo \& Sen (2005)) are among the many applications of stochastic integer programming. In that context the problem is to open a number of facilities that can be used to satisfy random client demand. This corresponds to allocating spare transformers that can be used to respond to random transformer failures. Our problem instances are far larger than what appears to be solvable exactly with current technology, where problem size is measured by the number of possible facility locations. For example, the largest instances solved in Ntaimo \& Sen (2005) have 10 and 15 possible facility locations whereas we solve instances with up to 71 candidate locations. Our problem becomes much larger when we add dimensions such as transformer type (e.g. one-phase vs. threephase), time of arrival (relevant to multistage applications) and other features (e.g. self- 
monitoring maintenance). It is clear from the CPU times reported in Ntaimo \& Sen (2005) that the number of possible facility locations has a decisive impact on run times. These findings are consistent with the results reported in Powell et al. (2004) (see also Topaloglu (2001)) which show that Benders decomposition becomes quite slow as the dimensionality of the second stage resource vector grows.

If we assume that there can be at most one failure and that we always meet that failure with a spare then our problem is equivalent to a generalization of the classic deterministic p-median problem given by Mirchandani (1990) and Labbé et al. (1995). This equivalence breaks down as soon as we allow more than one failure or if we allow that a failure is left unmet because the transfer cost is higher than the avoided congestion cost. Nevertheless we will make extensive use of the p-median model as a benchmark for our algorithms.

Several papers in the electric power literature (Chowdhury \& Koval (2005), Li et al. (1999), Kogan et al. (1996)) present techniques to determine the number of transformer spares to be held. None of these papers considers the issue of spare location which is central to our approach.

Prior research in stochastic resource allocation problems has shown that separable, piecewise linear function approximations work extremely well for two-stage resource allocation problems (see Godfrey \& Powell (2002), Powell et al. (2004), Topaloglu \& Powell (2006)), providing near-optimal results while scaling easily to very large scale, multistage applications (see Powell \& Topaloglu (2004)). This work suggests that this strategy might be very practical for the problem of managing spare transformers. However, all of this work was in the context of managing large fleets of vehicles which exhibits very different problem characteristics. The problem of allocating spare transformers, where there may be a half dozen spares spread among 70 locations, is quite different and it was not clear that the same strategies would work (our experiments confirmed this).

Our paper makes three contributions. 1) We present an approximate dynamic programming algorithm that provides very good solutions to realistic instances of the two-stage spare transformer allocation problem. 2) In the context of approximate dynamic programming, we illustrate the shortcomings of standard linear value function approximations when the true 
value function is not separable. We introduce new value function approximations that take into account this nonseparability. 3) We contribute insights into spare transformer allocation issues of practical interest. The location and number of spares for the PJM system are important questions as are the value of sharing spares among TOs, the role of ordering lead times, and the influence of transformer transportation costs.

Section 2 contains the basic notation and the model formulation of the spare transformer allocation problem. Section 3 introduces the algorithmic framework that we adopt to solve the problem. The main ingredients of our approach are suitable value function approximations which we present in section 4. We validate our algorithmic approach and apply it to PJM's system performing a series of computational experiments which are described in section 5 . The results of the experiments are presented in section 6. We state conclusions and directions for further research in section 7 .

\section{Model Formulation}

Each transformer that is in operation in the electric grid belongs to a transformer bank. A bank is a set of transformers that together handle all three power phases. Each bank consists of either a single three-phase transformer, or three single phase transformers. Failures are modeled at the level of banks since if a single-phase transformer fails, the entire bank has to be shut down. Once the failed transformer is replaced the bank is brought back online.

If a bank fails, then electricity has to be routed through other transformers. However, there is a limit to how much electricity can be routed along specific links in the network. As a result, if a bank fails, then it may be necessary to obtain power from a utility that may be more expensive, but whose location allows electricity to be routed through paths that have available capacity. The additional cost of acquiring power from a more expensive utility due to capacity constraints (resulting from a failed transformer) is referred to as congestion costs. The goal of our problem is to minimize the total expected cost incurred as a result of bank failures.

Transformers and banks share the same attribute space $\mathcal{A}$. For the purposes of this paper 
an element $a \in \mathcal{A}$ is a three-dimensional vector indicating a bank identifier, the location, and the transmission owner. An example for an element $a \in \mathcal{A}$ is

$$
a=\left(\begin{array}{c}
a_{1} \\
a_{2} \\
a_{3}
\end{array}\right)=\left(\begin{array}{c}
1 \\
\text { Branchburg } \\
\text { PSEG }
\end{array}\right)
$$

In this example PSEG is the transmission owner. The Branchburg substation belongs to PSEG's service area and 1 identifies the bank within the Branchburg substation. There are 71 $500 / 230 \mathrm{kV}$ banks in PJM's system and that is the largest attribute space we consider in our numerical work. The notation and our model are completely general and equipped to include other transformer attributes. We are not using any technical transformer attributes as part of our attribute vector because we assume a universal type of transformer spare - one that from a technical standpoint can be used to replace any failed transformer anywhere in the system. However, our methodology can be naturally extended to handle these additional attributes.

The state of the system is defined as

$$
\begin{aligned}
R_{t a}= & \text { number of spares with attribute } a \text { at time } t \in\{0,1\} \text { after } \\
& \text { the time } t \text { decisions are made. } \\
R_{t}= & \left(R_{t a}\right)_{a \in \mathcal{A}} \\
= & \text { resource state vector. }
\end{aligned}
$$

We denote a decision by $d \in \mathcal{D}$ where $\mathcal{D}$ is the set of decisions that can be used to act on a transformer. The set $\mathcal{D}$ contains three decision types, i.e. $\mathcal{D}=\mathcal{D}^{\text {buy }} \cup \mathcal{D}^{\text {move }} \cup d^{\emptyset}$ where the subsets are defined as follows:

$$
\begin{aligned}
\mathcal{D}^{\text {buy }}= & \text { decision to buy a spare transformer. There is one element } \\
& \text { in this set for every possible purchasing source, i.e. manu- } \\
& \text { facturer or manufacturing facility. } \\
\mathcal{D}^{\text {move }}= & \text { decisions to move a spare to a particular failure site and } \\
& \text { use it to meet the failure. There is one decision for every } \\
& \text { possible failure site, i.e. for every element of } \mathcal{A} .
\end{aligned}
$$




$$
d^{\emptyset}=\text { decision to hold a transformer (do nothing). }
$$

Using the set notation introduced above we define the generic decision variables $x_{t}=$ $\left(x_{t a d}\right)_{a \in \mathcal{A}, d \in \mathcal{D}}$ where

$$
\begin{aligned}
x_{t a d}= & \text { the number of transformers with attribute } a \text { acted on with } \\
& \text { decision } d \text { at time } t \text { and } \\
c_{t a d}= & \text { the cost parameter associated with } x_{t a d} .
\end{aligned}
$$

The parameter $B$ specifies the number of spares that we want to maintain, which we assume is determined by a policy decision made by management. In our model we fix the number of spares and concentrate on the location decisions. However, in our numerical work we run the model for different values of $B$ and can thereby gain insight into the optimal number of spares as well.

Randomness is introduced in our model via the $0 / 1$ random variables $\hat{W}_{1}=\left(\hat{W}_{1 a}\right)_{a \in \mathcal{A}}$ where $\hat{W}_{1 a}$ indicates if transformer bank $a$ failed in time period 1 . The model underlying $\hat{W}_{1}$ is the finite probability space $(\Omega, \mathcal{F}, \mathbb{P})$ where $\Omega$ is the set of all possible failure scenarios, $\omega, \mathcal{F}$ is the discrete $\sigma$-algebra on $\Omega$, and $\mathbb{P}$ is the probability measure that assigns a given probability to each element $\omega \in \Omega$.

$V_{0}\left(R_{0}\right)$ is the value function, which is the expectation of the second stage costs as a function of the information of the first stage. We can now present the optimization model. The problem is to find

$$
\min _{x_{0}}\left\{\sum_{a \in \mathcal{A}} \sum_{d \in \mathcal{D}^{b u y}} c_{0 a d} x_{0 a d}+V_{0}\left(R_{0}\right)\right\}
$$

where

$$
V_{0}\left(R_{0}\right)=\mathbb{E}\left\{\min _{x_{1}}\left[\sum_{a \in \mathcal{A}}\left(\sum_{d \in \mathcal{D}^{\text {move }}} c_{1 a d} x_{1 a d}+c_{1 a d^{\emptyset}} x_{1 a d^{\emptyset}}\right)\right] \mid R_{0}\right\}
$$


subject to:

$$
\begin{aligned}
& \sum_{a \in \mathcal{A}} \sum_{d \in \mathcal{D}^{\text {buy }}} x_{0 a d}=B \quad \forall a \\
& \sum_{d \in \mathcal{D}^{\text {buy }}} x_{0 a d}-R_{0 a}=0 \quad \forall a \\
& x_{0 a d} \in\{0,1\} \quad \forall a, d \in \mathcal{D}^{b u y} \\
& \sum_{d \in \mathcal{D}^{\text {move }} \cup d^{\emptyset}} x_{1 a d}(\omega)-R_{0 a}=0 \quad \forall a, \omega \\
& x_{1 a d}(\omega) \leq \hat{W}_{1 a}(\omega) \quad \forall a, d \in \mathcal{D}^{\text {move }}, \omega \\
& x_{1 a d}(\omega) \in\{0,1\} \quad \forall a, d \in \mathcal{D}^{\text {move }} \cup d^{\emptyset}, \omega .
\end{aligned}
$$

In this model formulation a movement decision implies a) that the spare is moved to a failure site and b) that the spare is used at the destination location to replace a failed transformer. Therefore, the coefficients $c_{1 a d}$ for $d \in \mathcal{D}^{\text {move }}$ contain two components: the cost associated with the movement, which we call transfer cost, and the avoided congestion costs due to the replacement of the failed transformer. Thus, this model minimizes the sum of transformer purchase costs, transfer costs, avoided congestion costs, and inventory holding costs of spares.

Equation (3) is the budget constraint that fixes the number of spares to be acquired. Equation (4) defines the resource state. The acquisition variables are binary as expressed in equation (5). Equation (6) ensures flow conservation in the first stage. Equation (7) states that a spare can only be used to meet a failure if in fact a failure occurred.

\section{Basic Algorithm}

Solving model (1)-(8) directly is computationally infeasible for problems of realistic size. The experimental evidence in Louveaux \& Peeters (1992), Laporte et al. (1994), and Ntaimo \& Sen (2005) shows this for integer stochastic programming based algorithms. Interestingly, the results in Ntaimo \& Sen (2005) suggest that computational difficulties do not necessarily arise

with a large sample space but with a high dimensional first-stage decision vector $x_{0}=\left(x_{0 a}\right)_{a \in \mathcal{A}}$. 
Classic stochastic dynamic programming (Puterman (1994) ) is also out of the question as a solution approach as it suffers from the well-known "curse of dimensionality" (Powell (2007)) caused by a large action space $\left(x_{0}\right)$, a large state space $\left(R_{0}\right)$, and a large sample space $(\Omega)$.

In order to address these computational problems we turn to approximate dynamic programming (ADP). This set of techniques has recently proven useful in finding very good approximate solutions for large-scale multi-period resource allocation problems (Powell et al. (2002), Topaloglu \& Powell (2006)). Powell \& Van Roy (2004) give an introduction to ADP in the context of resource allocation problems.

The central idea in ADP is to replace the value function $V\left(R_{0}\right)$ with an approximation $\bar{V}\left(R_{0}\right)$ that depends only on $R_{0}$ - the information known at time zero. In order to illustrate the main idea let us for the moment assume a linear value function approximation

$$
\bar{V}_{0}\left(R_{0}\right)=\sum_{a \in \mathcal{A}} \bar{v}_{a} R_{0 a}
$$

where the $\bar{v}_{a}$ are estimates of the unknown parameters $v_{a}$ which - in this case - can be interpreted as the marginal value of a resource with attribute $a$.

Using this approximation, the model becomes:

$$
\min _{x_{0}}\left\{\sum_{a \in \mathcal{A}} \sum_{d \in \mathcal{D}^{b u y}} c_{0 a d} x_{0 a d}+\sum_{a \in \mathcal{A}} \bar{v}_{a} R_{0 a}\right\}
$$

subject to:

$$
\begin{aligned}
\sum_{a \in \mathcal{A}} \sum_{d \in \mathcal{D}^{b u y}} x_{0 a d} & =B \quad \forall a \\
\sum_{d \in \mathcal{D}^{\text {buy }}} x_{0 a d}-R_{0 a} & =0 \quad \forall a \\
0 \leq x_{0 a d} & \leq 1 \quad \forall a, d \in \mathcal{D}^{\text {buy }}
\end{aligned}
$$

As we can see the model is now radically simplified. The random variable $\hat{W}_{1}$, the expectation with respect to $\hat{W}_{1}$, and the random recourse decisions $x_{1}$ have been eliminated from the model as the entire second stage has been replaced by an approximation. The resulting 
approximate model can be easily solved using commercially available optimization software such as CPLEX.

Using a value function approximation (VFA) comes at a price. Once a particular functional form of the approximation is chosen the challenge lies in the estimation of the parameters $\bar{v}_{a}$. In this sense we have replaced an optimization problem with an estimation problem.

In the following we give a high level description of an ADP algorithm that uses stochastic gradients to perform this estimation. For illustrative purposes we continue to use the linear VFA of equation (9). Detailed algorithms using different value function approximations are given in section 4 .

The core of the algorithm has three steps that are iterated $N$ times. In our notation index $n$ denotes the iteration. The first step consists of solving the approximate problem (10)-(13). In the second step the algorithm takes a failure sample $\hat{W}_{1}\left(\omega^{n}\right)$ and determines sample gradients of the true value function with respect to the $R_{0 a}^{n}$. These sample gradients are used to obtain a sample realization $\hat{v}_{a}^{n}$ of the parameter value. Sampling failures and solving the second stage problem for that sample realization prepares the ground for the gradient calculation. Let

$$
\begin{aligned}
m(d)= & \text { the attribute resulting from modifying a resource with de- } \\
& \text { cision } d \text {. }
\end{aligned}
$$

The second stage problem is to find

$$
\hat{F}\left(R_{0}^{n}, \hat{W}_{1}\left(\omega^{n}\right)\right)=\min _{x_{1}}\left\{\sum_{a \in \mathcal{A}}\left(\sum_{d \in \mathcal{D}^{\text {move }}} c_{a d} x_{1 a d}+c_{a d^{\emptyset}} x_{1 a d^{\emptyset}}\right)\right\}
$$

subject to:

$$
\begin{aligned}
\sum_{d \in \mathcal{D}^{\text {move } \cup d^{\emptyset}}} x_{1 a d} & =R_{0 a}^{n} \quad \forall a \\
\sum_{a^{\prime} \in \mathcal{A}} x_{1 a^{\prime} d} & \leq \hat{W}_{1, m(d)}\left(\omega^{n}\right) \quad \forall d \in \mathcal{D}^{\text {move }} \\
0 \leq x_{1 a d} & \leq 1 \quad \forall a, d \in \mathcal{D}^{\text {move }} \cup d^{\emptyset}
\end{aligned}
$$


Note that in this model $R_{0}^{n}$ is determined by $x_{0}^{n}$, the solution to the first-stage problem. $\hat{F}$ is also a function of the failure sample $\hat{W}_{1}\left(\omega^{n}\right)$. For the sake of notational simplicity we will omit $\hat{W}_{1}\left(\omega^{n}\right)$ as an argument of $\hat{F}$ henceforth. Suppose now that $R_{0 a}^{n}$ is 1 for a particular $a$. Then the sample gradient of the true value function with respect $R_{0 a}^{n}$ is a left gradient of the form:

$$
\hat{v}_{a}^{n}=\hat{F}\left(R_{0}^{n}\right)-\hat{F}\left(R_{0}^{n}-e_{a}\right)
$$

where $e_{a}$ is a vector of zeros with a one at element $a$.

In the third step of the algorithm we use the newly obtained sample realization $\hat{v}_{a}^{n}$ to update our estimate $\bar{v}_{a}^{n}$. The updating follows the general formula

$$
\bar{v}_{a}^{n}=\left(1-\alpha_{n-1}\right) \bar{v}_{a}^{n-1}+\alpha_{n-1} \hat{v}_{a}^{n}
$$

where $\alpha_{n-1}$ is the step size in iteration $n$.

Step 0: Initialize $\bar{V}_{0}^{0}$ and set $n=1$.

Step 1: Solve the approximate problem to get $x_{0}^{n}$ and $R_{0}^{n}$. If $n=N+1$ stop. $x_{0}^{N+1}$ is the solution.

Step 2: Sample $\hat{W}_{1}\left(\omega^{n}\right)$ and determine sample realizations of VFA parameters using sample gradients.

Step 3: Update VFA parameter estimates as in equation (19). Increment $n$ and go to Step 1.

Figure 1: General ADP algorithm for the spare allocation problem

Figure 1 lists the steps of the ADP algorithm. The more detailed algorithms of section 4 are variations of this general approach. Using this algorithmic approach successfully hinges on finding good value function approximations. This means finding appropriate functional forms that can be estimated with a reasonable amount of effort. This is the task of the next section. 


\section{Value Function Approximations}

In this section we present different value function approximations. We start with the linear VFA as a natural starting point and progress to somewhat more sophisticated approximations that address the limitations of the linear approach.

\subsection{Linear Approximation}

We have used a linear value function approximation as an example before and repeat the definition here for convenience. The linear approximation has the following form:

$$
\bar{V}_{0}\left(R_{0}\right)=\sum_{a \in \mathcal{A}} \bar{v}_{a} R_{0 a}
$$

A linear VFA has been shown to be effective in the context of certain types of resource allocation problems (see Powell et al. $(2002)$ ). It also has a very intuitive interpretation: $\bar{v}_{a}$ is the estimate of the value of a transformer with attributes $a$. It is intuitively clear that spares can have different values. For example, a spare in a central network location might have a higher value than a spare in a remote network location.

The use of this approximation leads to the approximate problem $(10)-(13)$. If $R_{0 a}^{n}$ is 1 then the sample gradient of the true value function with respect $R_{0 a}^{n}$ is a left gradient of the form:

$$
\hat{v}_{a}^{l e f t, n}=\hat{F}\left(R_{0}^{n}\right)-\hat{F}\left(R_{0}^{n}-e_{a}\right)
$$

If $R_{0 a}^{n}$ is 0 then the sample gradient is a right gradient. The right gradients require special consideration. If we just added a spare $a$ to calculate $\hat{F}\left(R_{0}^{n}+e_{a}\right)$ then we would have $B+1$

spares in the system. The right gradient $\hat{F}\left(R_{0}^{n}+e_{a}\right)-\hat{F}\left(R_{0}^{n}\right)$ would be the marginal value of spare $a$ if it was the $B+1^{\text {st }}$ spare. Clearly, this gradient is not the best choice if we are restricted to placing $B$ spares. The gradient is influenced by two effects, namely by the attributes of the spare (location effect) and by the fact that we have $B+1$ spares (quantity effect). But if we are placing $B$ transformers we are only interested in the location effect. 
To address this we calculate the right gradients as

$$
\hat{v}_{a}^{r i g h t, n}=\hat{F}\left(R_{0}^{n}-e_{a^{*}}+e_{a}\right)-\hat{F}\left(R_{0}^{n}-e_{a^{*}}\right)
$$

where $a^{*}$ is the marginal spare, i.e. the least valuable of all the $B$ selected spares. Now the right gradients have the same interpretation as do left gradients. They measure the marginal value of $a$ as the $B^{\text {th }}$ spare.

The updating equations for the parameter estimates are

$$
\bar{v}_{a}^{n}= \begin{cases}\left(1-\alpha_{n-1}\right) \bar{v}_{a}^{n-1}+\alpha_{n-1} \hat{v}_{a}^{r i g h t, n} & \text { if } R_{0 a}^{n}=0 \\ \left(1-\alpha_{n-1}\right) \bar{v}_{a}^{n-1}+\alpha_{n-1} \hat{v}_{a}^{l e f t, n} & \text { if } R_{0 a}^{n}=1 .\end{cases}
$$

Figure 2 gives a step by step view of the ADP algorithm using a linear VFA.

Step 0: Initialize $\bar{V}_{0}^{0}$ and set $n=1$.

Step 1: Solve 10p-13 to get $x_{0}^{n}$ and $R_{0}^{n}$. If $n=N+1$ stop. $x_{0}^{N+1}$ is the solution.

Step 2: Sample $\hat{W}_{1}\left(\omega^{n}\right)$ and determine gradients $\hat{v}_{a}^{n} \forall a \in \mathcal{A}$ as in 21, and 22.

Step 3: Calculate $\bar{v}_{a}^{n} \forall a \in \mathcal{A}$ as in 23).

Increment $n$ and go to Step 1.

Figure 2: Spare allocation algorithm with linear VFA

Our numerical experiments will provide detailed insight into the solution quality obtained using a linear VFA. We expose its main limitation here to motivate the presentation of our other approximations. Using the linear VFA the ADP algorithm produces solutions as shown in figure 3, which depicts part of PJM's service area. Shown is the spare allocation for part of PJM's service area. Overlapping circles indicate substation locations with multiple spares.

The algorithm correctly identifies good spare locations, but when a location has multiple banks, it often allocates multiple spares leading to bunching.

The reason for this behavior is the separability of the linear VFA with respect to the elements of $R_{0}$. Separability means that the value of a spare transformer with certain attributes is assumed to be independent of the spare allocation in the rest of the network. This is certainly not true. For example, the value of the first spare in a location is very different from 


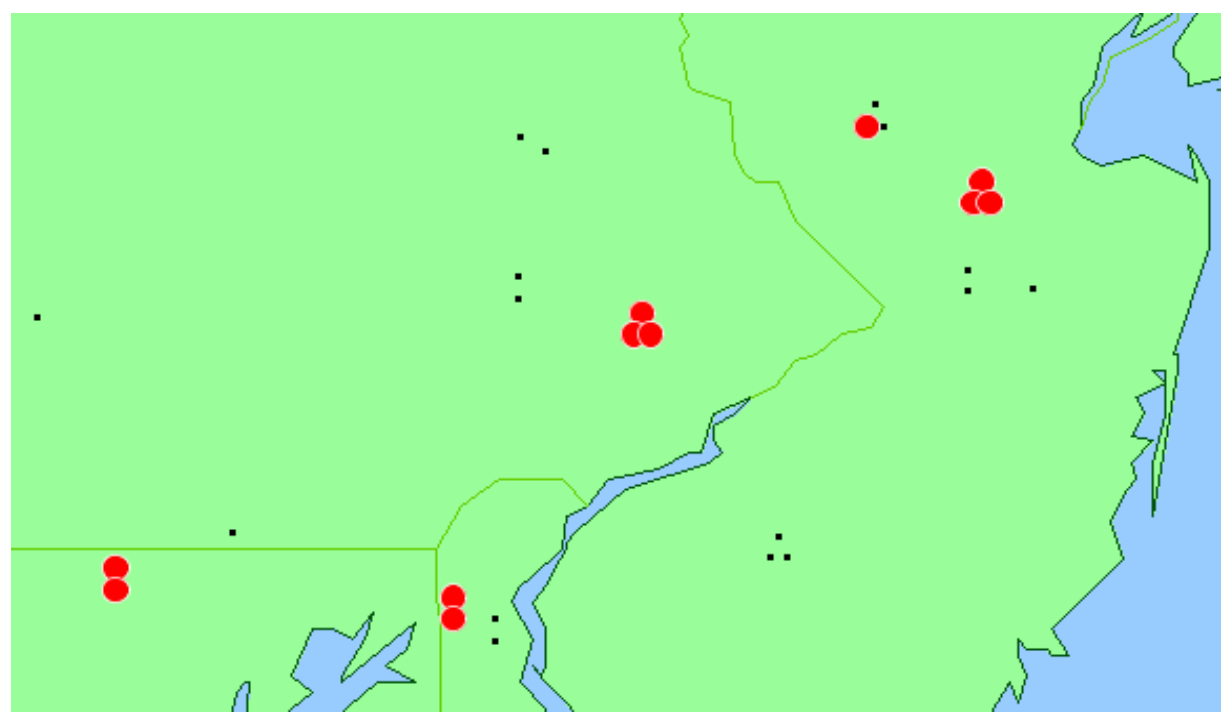

Figure 3: Partial solution obtained using a linear VFA. The small spots are bank locations which are slightly perturbed to show multiple banks in the same location. Transformer spares - indicated by circles - are bunched in "good" locations with multiple banks.

the value of the second or the third. Clearly, the true value function has non-separable effects with respect to the elements of $R_{0}$. In the following we present two approximation strategies that incorporate this non-separability.

\subsection{Quadratic Approximation}

We wish to find VFAs that allow us to make the contribution of a spare dependent on the remaining spare allocation. One way to introduce such dependence is by considering pairs $\left\{a, a^{\prime}\right\}$ of spare attributes. Let us define the relevant set of such pairs as

$$
\mathcal{P}=\text { set of unordered attribute pairs }\left\{\left\{a, a^{\prime}\right\} \mid a \in \mathcal{A}, a^{\prime} \in \mathcal{A}, a \neq a^{\prime}\right\}
$$

We assume the following model based on attribute pairs:

$$
\hat{F}\left(R_{0}\right)=\sum_{\left\{a, a^{\prime}\right\} \in \mathcal{P}} \beta_{a a^{\prime}}^{00}{ }_{a a^{\prime}}^{00}+\beta_{a a^{\prime}}^{10} 1_{a a^{\prime}}^{10}+\beta_{a a^{\prime}}^{01} 1_{a a^{\prime}}^{01}+\beta_{a a^{\prime}}^{11} 1_{a a^{\prime}}^{11}+\epsilon
$$


where we write $1_{a a^{\prime}}^{00}$ for $1_{\left\{R_{0 a}=0, R_{0 a^{\prime}}=0\right\}}$ and where $\epsilon$ stands for i.i.d. error terms with expectation zero. Note that in this model every pair of attributes $\left\{a, a^{\prime}\right\}$ contributes to the value of the allocation with one $\beta$ that depends on the values of $R_{0 a}$ and $R_{0 a^{\prime}}$. This is fundamentally different from the linear approximation where the value of an allocation is determined by looking at single attributes rather than attribute pairs. By replacing the indicator functions in equation $(24)$ we obtain

$$
\begin{aligned}
& \hat{F}\left(R_{0}\right)= \\
& \quad \sum_{\left\{a, a^{\prime}\right\} \in \mathcal{P}} \beta_{a a^{\prime}}^{00}\left(1-R_{0 a}\right)\left(1-R_{0 a^{\prime}}\right)+\beta_{a a^{\prime}}^{10} R_{0 a}\left(1-R_{0 a^{\prime}}\right)+\beta_{a a^{\prime}}^{01}\left(1-R_{0 a}\right) R_{0 a^{\prime}}+\beta_{a a^{\prime}}^{11} R_{0 a} R_{0 a^{\prime}}+\epsilon
\end{aligned}
$$

Multiplying out and collecting terms results in:

$$
\hat{F}\left(R_{0}\right)=K+\sum_{a \in \mathcal{A}} \theta_{a} R_{0 a}+\sum_{\left\{a, a^{\prime}\right\} \in \mathcal{P}} \theta_{a a^{\prime}} R_{0 a} R_{0 a^{\prime}}+\epsilon
$$

where

$$
\begin{aligned}
K & =\sum_{\left\{a, a^{\prime}\right\} \in \mathcal{P}} \beta_{a a^{\prime}}^{00} \\
\theta_{a} & =\sum_{a^{\prime} \neq a} \beta_{a a^{\prime}}^{10}-\beta_{a a^{\prime}}^{00} \\
\theta_{a a^{\prime}} & =\beta_{a a^{\prime}}^{00}-\beta_{a a^{\prime}}^{10}-\beta_{a a^{\prime}}^{01}+\beta_{a a^{\prime}}^{11}
\end{aligned}
$$

This model gives rise to the value function approximation

$$
\bar{V}_{0}\left(R_{0}\right)=K+\sum_{a \in \mathcal{A}} \bar{\theta}_{a} R_{0 a}+\sum_{\left\{a, a^{\prime}\right\} \in \mathcal{P}} \bar{\theta}_{a a^{\prime}} R_{0 a} R_{0 a^{\prime}}
$$

We see that our assumption leads to a quadratic non-separable value function approximation. The $\bar{\theta}_{a}$ can not be interpreted as the value of spare $a$ because the value of spare $a$ depends on other allocations across the network. However, the $\bar{\theta}_{a a^{\prime}}$ have an intuitive interpretation. $\bar{\theta}_{a a^{\prime}}$ is a penalty for allocating spares to $a$ and $a^{\prime}$ simultaneously. The higher $\bar{\theta}_{a a^{\prime}}$ the less desirable it is to have a spare in both places. 
We now have to show that we can solve the resulting approximate problem and that we can estimate the parameters of equation (26). We first show how to solve the approximate problem, which is to find

$$
\min _{x_{0}}\left\{\sum_{a \in \mathcal{A}} \sum_{d \in \mathcal{D}^{b u y}} c_{0 a d} x_{0 a d}+\sum_{a \in \mathcal{A}} \bar{\theta}_{a} R_{0 a}+\sum_{\left\{a, a^{\prime}\right\} \in \mathcal{P}} \bar{\theta}_{a a^{\prime}} R_{0 a} R_{0 a^{\prime}}\right\}
$$

subject to:

$$
\begin{aligned}
\sum_{a \in \mathcal{A}} \sum_{d \in \mathcal{D}^{\text {buy }}} x_{0 a d} & =B \quad \forall a \\
\sum_{d \in \mathcal{D}^{\text {buy }}} x_{0 a d}-R_{0 a} & =0 \quad \forall a \\
x_{0 a d} & \in\{0,1\} \quad \forall a, d \in \mathcal{D}^{b u y} .
\end{aligned}
$$

Note that we omit the constant $K$ in the objective function because it does not affect the solution. This model is a quadratic mixed 0-1 program which is much harder to solve than the linear model (10)-(13). In order to facilitate the computational treatment of (27)-(30) we linearize it to obtain an equivalent linear mixed 0-1 program (see Helmberg (2000)). We define $y_{a a^{\prime}}=R_{0 a} R_{0 a^{\prime}}$. The linearized approximate problem then is to find

$$
\min _{x_{0}}\left\{\sum_{a \in \mathcal{A}} \sum_{d \in \mathcal{D}^{b u y}} c_{0 a d} x_{0 a d}+\sum_{a \in \mathcal{A}} \bar{\theta}_{a} R_{0 a}+\sum_{\left\{a, a^{\prime}\right\} \in \mathcal{P}} \bar{\theta}_{a a^{\prime}} y_{a a^{\prime}}\right\}
$$

subject to:

$$
\begin{aligned}
R_{0 a}+R_{0 a^{\prime}}-y_{a a^{\prime}} & \leq 1 \quad \forall\left\{a, a^{\prime}\right\} \in \mathcal{P} \\
y_{a a^{\prime}} & \leq R_{0 a} \quad \forall\left\{a, a^{\prime}\right\} \in \mathcal{P} \\
y_{a a^{\prime}} & \leq R_{0 a^{\prime}} \quad \forall\left\{a, a^{\prime}\right\} \in \mathcal{P} \\
y_{a a^{\prime}} & \geq 0 \quad \forall\left\{a, a^{\prime}\right\} \in \mathcal{P} \\
x_{0} & \in \mathcal{X}_{0}
\end{aligned}
$$

where the last constraint expresses equations 28)-(30) from before. Note that only the $x_{0 a b}$ need a binary constraint; the $y_{a a^{\prime}}$ do not. 
We now turn to the issue of estimating the parameters of the quadratic approximation. Below we show how to obtain sample realizations of the parameters $\theta_{a}$ and $\theta_{a a^{\prime}}$. In these computations we use second stage objective function values $\hat{F}$, where the resource state vector is perturbed around the initial solution $R_{0}$. Let, for example, $R_{0 a}=0$ and $R_{0 a^{\prime}}=0$ be part of the initial solution. Then

$$
\begin{aligned}
\hat{F}\left(R_{0}\right) & =\hat{F}\left(R_{0 a^{(1)}}, R_{0 a^{(2)}}, \ldots, R_{0 a}, \ldots, R_{0 a^{\prime}}, \ldots, R_{0 a^{|\mathcal{A}|}}\right) \\
& =\hat{F}\left(R_{0 a^{(1)}}, R_{0 a^{(2)}}, \ldots, 0, \ldots, 0, \ldots, R_{0 a|\mathcal{A}|}\right)
\end{aligned}
$$

We define

$$
\hat{F}_{a a^{\prime}}^{11}=\hat{F}\left(R_{0 a^{(1)}}, R_{0 a^{(2)}}, \ldots, 1, \ldots, 1, \ldots, R_{0 a|\mathcal{A}|}\right)
$$

For the example case $R_{0 a}=0$ and $R_{0 a^{\prime}}=0$ this corresponds to the perturbation

$$
\hat{F}_{a a^{\prime}}^{11}=\hat{F}\left(R_{0}+e_{a}+e_{a^{\prime}}\right)
$$

$\hat{F}_{a a^{\prime}}^{10}, \hat{F}_{a a^{\prime}}^{01}$, and $\hat{F}_{a a^{\prime}}^{00}$ are defined analogously. Let $\mathcal{A}_{1}=\left\{a^{\prime \prime} \in \mathcal{A} \backslash a \mid R_{0 a^{\prime \prime}}=1\right\}$ which is the set of all transformer banks that are different from $a$ and have a spare. We define

$$
\begin{aligned}
& \hat{\theta}_{a}=\left\{\begin{array}{l}
\sum_{a^{\prime \prime} \in \mathcal{A}_{1}}\left(\hat{F}_{a a^{\prime \prime}}^{10}-\hat{F}_{a a^{\prime \prime}}^{00}\right)-\left(\left|\mathcal{A}_{1}\right|-1\right)\left(\hat{F}\left(R_{0}+e_{a}\right)-\hat{F}\left(R_{0}\right)\right), \\
\sum_{a^{\prime \prime} \in \mathcal{A}_{1}}\left(\hat{F}_{a a^{\prime \prime}}^{10}-\hat{F}_{a a^{\prime \prime}}^{00}\right)-\left(\left|\mathcal{A}_{1}\right|-1\right)\left(\hat{F}\left(R_{0}\right)-\hat{F}\left(R_{0}-e_{a}\right)\right), \quad \text { for } R_{0 a}=0,
\end{array}\right. \\
& \hat{\theta}_{a a^{\prime}}=\hat{F}_{a a^{\prime}}^{11}-\hat{F}_{a a^{\prime}}^{10}-\hat{F}_{a a^{\prime}}^{01}+\hat{F}_{a a^{\prime}}^{00} .
\end{aligned}
$$

In iteration $n$ the $\hat{\theta}_{a}^{n}$ and $\hat{\theta}_{a a^{\prime}}^{n}$ are calculated and smoothed into the current estimates in the standard way:

$$
\begin{aligned}
\bar{\theta}_{a}^{n} & =\left(1-\alpha_{n-1}\right) \bar{\theta}_{a}^{n-1}+\alpha_{n-1} \hat{\theta}_{a}^{n} \\
\bar{\theta}_{a a^{\prime}}^{n} & =\left(1-\alpha_{n-1}\right) \bar{\theta}_{a a^{\prime}}^{n-1}+\alpha_{n-1} \hat{\theta}_{a a^{\prime}}^{n}
\end{aligned}
$$


Step 0: Initialize $\bar{V}_{0}$ to $\bar{V}_{0}^{0}$. Choose a steps size rule $\alpha_{n}$ such that $\alpha_{0}=1$ and $0 \leq \alpha_{n} \leq 1$ for $n=1, \ldots, N$. Set $n=1$.

Step 1: Solve 317- -36 to get $x_{0}^{n}$ and $R_{0}^{n}$. If $n=N+1$ stop. $x_{0}^{N+1}$ is the solution.

Step 2: Sample $\hat{W}_{1}\left(\omega^{n}\right)$ and determine sample realizations $\hat{\theta}_{a}^{n} \quad \forall a \in \mathcal{A}$ and $\hat{\theta}_{a a^{\prime}}^{n} \forall\left\{a, a^{\prime}\right\} \in \mathcal{P}$ as in 38 and (37).

Step 3: Calculate $\bar{\theta}_{a}^{n} \forall a \in \mathcal{A}$ and $\bar{\theta}_{a a^{\prime}}^{n} \quad \forall\left\{a, a^{\prime}\right\} \in \mathcal{P}$ as in equations 39 and 40 .

Increment $n$ and go to step 1.

Figure 4: Spare allocation algorithm with quadratic VFA

The complete ADP algorithm using the quadratic value function approximation is given in figure 4 .

With the formulas for $\hat{\theta}_{a}$ and $\hat{\theta}_{a a^{\prime}}$ we can correctly separate the effect of the variable $R_{0 a}$ from the effects of the cross terms $R_{0 a} R_{0 a^{\prime}}$. The following proposition makes this statement precise.

Proposition. Assume the model of equation (25) and let $\theta_{a}$ and $\theta_{a a^{\prime}}$ be estimated with the procedure in figure 4. Then $\bar{\theta}_{a}^{n}$ and $\bar{\theta}_{a a^{\prime}}^{n}$ are unbiased estimators of $\theta_{a}$ and $\theta_{a a^{\prime}}$ respectively for all $a \in \mathcal{A},\left\{a, a^{\prime}\right\} \in \mathcal{P}$ and $n=1, \ldots, N$.

Proof: We start by showing that $\hat{\theta}_{a}$ is an unbiased estimator of $\theta_{a}$ for all values of $R_{0}$. We use equation (37) to calculate $\hat{\theta}_{a}$ and show the proof for $R_{0 a}=0$. The case $R_{0 a}=1$ follows the same arguments. Pick $a^{\prime \prime} \in \mathcal{A}_{1}$.

$$
\begin{aligned}
\hat{F}_{a a^{\prime \prime}}^{10}-\hat{F}_{a a^{\prime \prime}}^{00} & =\hat{F}\left(R_{0}+e_{a}-e_{a^{\prime \prime}}\right)-\hat{F}\left(R_{0}-e_{a^{\prime \prime}}\right) \\
& =\theta_{a}+\sum_{a^{*} \in \mathcal{A}_{1} \backslash a^{\prime}} \theta_{a a^{*}}+\epsilon^{*}
\end{aligned}
$$

where $\epsilon^{*}$ is a linear combination of error terms. Summing over all such $a^{\prime \prime}$ gives:

$$
\sum_{a^{\prime \prime} \in \mathcal{A}_{1}}\left(\hat{F}\left(R_{0}+e_{a}-e_{a^{\prime \prime}}\right)-\hat{F}\left(R_{0}-e_{a^{\prime \prime}}\right)\right)=\left|\mathcal{A}_{1}\right| \theta_{a}+\left(\left|\mathcal{A}_{1}\right|-1\right) \sum_{a^{*} \in \mathcal{A}_{1}} \theta_{a a^{*}}+\epsilon^{*}
$$

Furthermore,

$$
\hat{F}\left(R_{0}+e_{a}\right)-\hat{F}\left(R_{0}\right)=\theta_{a}+\sum_{a^{*} \in \mathcal{A}_{1}} \theta_{a a^{*}}+\epsilon^{*}
$$


Inserting (41) and (42) into equation (37) and canceling terms gives

$$
\hat{\theta}_{a}=\theta_{a}+\epsilon^{*}
$$

Note that according to $43 \hat{\theta}_{a}$ is not a function of $R_{0}$. Hence we can take expectations on both sides to get

$$
\mathbb{E}\left[\hat{\theta}_{a}\right]=\theta_{a}
$$

Having shown unbiasedness of $\hat{\theta}_{a}$ we proceed by induction. Let $n=1$. Since $\alpha_{0}=1$ we have by 39

$$
\bar{\theta}_{a}^{1}=\hat{\theta}_{a}^{1}
$$

and the unbiasedness of $\bar{\theta}_{a}^{1}$ follows from the unbiasedness of $\hat{\theta}_{a}^{1}$. Assume $\bar{\theta}_{a}^{n}$ is unbiased for $n=k-1$ and let $n=k$. We have by (39)

$$
\bar{\theta}_{a}^{k}=\left(1-\alpha_{k-1}\right) \bar{\theta}_{a}^{k-1}+\alpha_{k-1} \hat{\theta}_{a}^{k}
$$

Since $\bar{\theta}_{a}^{k-1}$ is unbiased by assumption and $\hat{\theta}_{a}^{k}$ is unbiased by 44 we obtain

$$
\mathbb{E}\left[\bar{\theta}_{a}^{k}\right]=\left(1-\alpha_{k-1}\right) \theta_{a}+\alpha_{k-1} \theta_{a}=\theta_{a}
$$

To show unbiasedness of $\bar{\theta}_{a a^{\prime}}^{n}$ we start by showing that $\hat{\theta}_{a a^{\prime}}$ is an unbiased estimator of $\theta_{a a^{\prime}}$ for all values of $R_{0}$. We use equation 38 to calculate $\hat{\theta}_{a a^{\prime}}$ and show the proof for the case where $R_{0 a}=1$ and $R_{0 a^{\prime}}=0$. The other three cases, i.e. $\left\{R_{0 a}, R_{0 a^{\prime}}\right\}=\{1,1\},\left\{R_{0 a}, R_{0 a^{\prime}}\right\}=\{0,1\}$, and $\left\{R_{0 a}, R_{0 a^{\prime}}\right\}=\{0,0\}$ follow the same arguments. Let $R_{0 a}=1$ and $R_{0 a^{\prime}}=0$. By equation 38

$$
\begin{aligned}
\hat{\theta}_{a a^{\prime}} & =\hat{F}\left(R_{0}+e_{a^{\prime}}\right)-\hat{F}\left(R_{0}\right)-\hat{F}\left(R_{0}-e_{a}+e_{a^{\prime}}\right)+\hat{F}\left(R_{0}-e_{a}\right) \\
& =\theta_{a^{\prime}}+\sum_{a^{\prime \prime} \in \mathcal{A}_{1} \cup a} \theta_{a^{\prime \prime} a^{\prime}}-\theta_{a^{\prime}}-\sum_{a^{\prime \prime} \in \mathcal{A}_{1}} \theta_{a^{\prime \prime} a^{\prime}}+\epsilon^{*} \\
& =\theta_{a a^{\prime}}+\epsilon^{*}
\end{aligned}
$$


where $\epsilon^{*}$ is a linear combination of error terms. Equation 45 shows that $\hat{\theta}_{a a^{\prime}}$ is not a function of $R_{0}$. Thus, taking expectations on both sides gives

$$
\mathbb{E}\left[\hat{\theta}_{a a^{\prime}}\right]=\theta_{a a^{\prime}}
$$

Unbiasedness of $\bar{\theta}_{a a^{\prime}}^{n}$ follows by induction.

\subsection{Piecewise Linear Approximation and Aggregation}

Another tool to incorporate non-separable effects in the VFA is aggregation. Aggregation means that the set $\mathcal{A}$ is partitioned into groups $\mathcal{A}_{1}, \mathcal{A}_{2}, \ldots, \mathcal{A}_{K}$ and that these groups are used in the VFA in a useful way. It is important to make clear that aggregation applies only to the VFA. We do not aggregate data elements such as failure probabilities or congestion costs, and we also do not aggregate purchasing decisions. The simulation of the system does not change and the optimization is affected only in so far as the VFA now has a different form.

Using the partition of $\mathcal{A}$ we define the aggregate resource state variable $R_{0}^{g}=\left(R_{0 k}^{g}\right)_{k=1,2, \ldots, K}$, where $R_{0 k}^{g}$ denotes the number of spares in group $k$. Formally, we have

$$
R_{0 k}^{g}=\sum_{a \in \mathcal{A}_{k}} R_{0 a}
$$

Using the aggregate resource state variable we introduce the aggregate VFA component $\bar{V}_{0}^{g}\left(R_{0}^{g}\right)$. The key advantage of aggregation is that $\bar{V}_{0}^{g}\left(R_{0}^{g}\right)$ can consist of nonlinear functions which allow us to capture the declining marginal value of spares in a group. If, for example, a group corresponds to a location then the aggregate VFA component can capture the fact that the first spare in a location is worth more than the second one which is worth more than the third and so forth.

We use piecewise linear, convex functions to approximate value functions of spares at an aggregated level. The usefulness of piecewise linear functions in VFAs was first documented in Godfrey \& Powell (2001) and Godfrey \& Powell (2002). Figure 5 illustrates the VFA with piecewise linear components. Note that the VFA contains both linear terms on the disaggregate level (i.e. the bank level) and aggregate piecewise linear terms. The existence of aggregate 


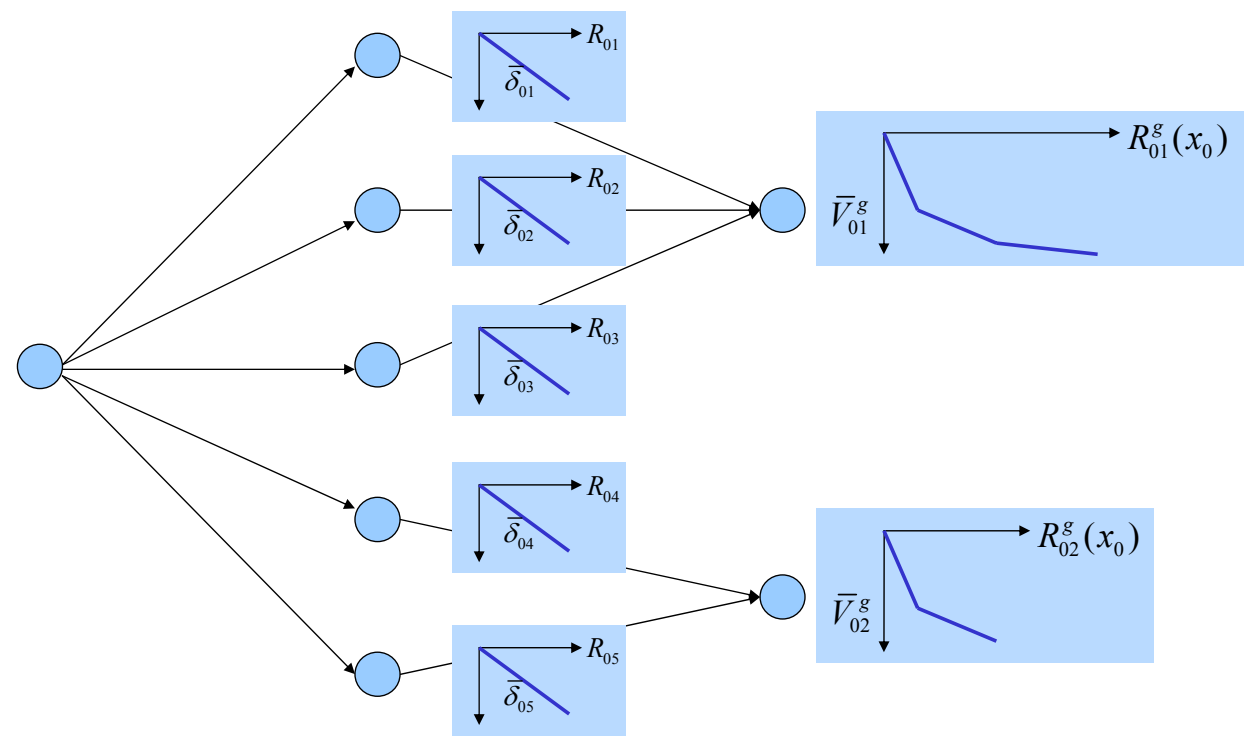

Figure 5: VFA with nonlinear components. The arcs on the left represent the purchasing decisions. The arcs in the middle are linear value function terms on the disaggregate level; on the right are piecewise linear aggregate VFA terms for every group.

and disaggregate VFA components comes from the fact that under the aggregation paradigm we look at the marginal value of a spare transformer, $v_{a}$, as containing two components. We can write for $a \in \mathcal{A}_{k}: v_{a}=v_{k}^{g}+\delta_{a}$. One value component, $v_{k}^{g}$, is derived from the group level and the other, $\delta_{a}$, is a correction term that differentiates spares in a group. Of course, none of these values is known, so we set out to describe how they can be estimated.

We define

$$
\begin{aligned}
y_{k r}= & \text { the flow on the } r^{t h} \text { segment of the piecewise linear function } \\
& \text { of group } k, \\
\bar{v}_{k r}^{g}= & \text { estimate of the slope of the } r^{t h} \text { segment of the piecewise } \\
& \text { linear function of group } k, \\
\bar{\delta}_{a}= & \text { slope of the disaggregate VFA term } a .
\end{aligned}
$$

The approximate problem is to find

$$
\min _{x_{0}}\left\{\sum_{a \in \mathcal{A}} \sum_{d \in \mathcal{D}^{b u y}} c_{0 a d} x_{0 a d}+\sum_{a \in \mathcal{A}} \bar{\delta}_{a} R_{0 a}+\sum_{k=1}^{K} \sum_{r=0}^{\left|\mathcal{A}_{k}\right|-1} \bar{v}_{k r}^{g} y_{k r}\right\}
$$


subject to:

$$
\begin{aligned}
\sum_{a \in \mathcal{A}_{k}} R_{0 a}-\sum_{r=0}^{\left|\mathcal{A}_{k}\right|-1} y_{k r} & =0 \forall k \\
0 \leq y_{k r} & \leq 1 \forall r, k \\
x_{0} & \in \mathcal{X}_{0}^{\prime}
\end{aligned}
$$

where $\mathcal{X}_{0}^{\prime}$ is the feasible region defined by (11)-(13). Note that the double sum in (47) is the aggregate VFA component $\bar{V}_{0}^{g}\left(R_{0}^{g}\right)$ and that 48 ensures flow conservation in the aggregate nodes.

The raw materials for the estimation of VFA slopes are the left and right sample gradients as defined in equations (21) and (22). The right gradient can be calculated if $R_{0 a}^{n}$ is 0 , the left gradient if $R_{0 a}^{n}$ is 1 . We define the sample gradient on the group level as:

$$
\begin{aligned}
\hat{v}_{k}^{g, r i g h t, n} & =\min _{a \in \mathcal{A}, R_{0 a}^{n}=0} \hat{v}_{a}^{\text {right }, n} \\
\hat{v}_{k}^{g, l e f t, n} & =\min _{a \in \mathcal{A}, R_{0 a}^{n}=1} \hat{v}_{a}^{l e f t, n} .
\end{aligned}
$$

The aggregate gradients are the minimum of the left and right gradients in the group. We find that using the minimum function to aggregate sample gradients produces much better solutions than using simple averages. Now we can calculate the sample correction terms $\hat{\delta}_{a}^{n}$ as the difference between the disaggregate sample gradient and the aggregate sample gradient:

$$
\hat{\delta}_{a}^{n}= \begin{cases}\hat{v}_{a}^{\text {right }, n}-\hat{v}_{k}^{g, \text { right }, n}, & \text { if } a \in \mathcal{A}_{k} \text { and } R_{0 a}^{n}=0 \\ \hat{v}_{a}^{\text {left,n}}-\hat{v}_{k}^{g, l e f t, n}, & \text { if } a \in \mathcal{A}_{k} \text { and } R_{0 a}^{n}=1\end{cases}
$$

It remains to be shown how to use $\hat{v}_{k}^{g, r i g h t, n}, \hat{v}_{k}^{g, l e f t, n}$, and $\hat{\delta}_{a}^{n}$ to update their respective VFA terms. The update of $\bar{\delta}_{a}^{n-1}$ follows the usual scheme

$$
\bar{\delta}_{a}^{n}=\left(1-\alpha_{n-1}\right) \bar{\delta}_{a}^{n-1}+\alpha_{n-1} \hat{\delta}_{a}^{n}
$$

Updating the piecewise linear VFA components $\bar{V}_{0 k}^{g}\left(R_{0 k}^{g}\right)$ requires a more involved procedure. Godfrey \& Powell (2001), Topaloglu \& Powell (2003), and Powell et al. (2004) propose 
different methods. Common to all methods is a preliminary step where $\hat{v}_{k}^{g, l e f t, n}$ is used to update the slope to the left of $R_{0 k}^{g n}$ and $\hat{v}_{k}^{g, r i g h t, n}$ is used to update the slope to the right of $R_{0 k}^{g n}$. The formula for this preliminary step is

$$
u_{k r}^{n}= \begin{cases}\left(1-\alpha_{n-1}\right) \bar{v}_{k r}^{g, n-1}+\alpha_{n-1} \hat{v}_{k}^{g, r i g h t, n} & \text { if } r=R_{0 k}^{g n}, \\ \left(1-\alpha_{n-1}\right) \bar{v}_{k r}^{g, n-1}+\alpha_{n-1} \hat{v}_{k}^{g, l e f t, n} & \text { if } r=R_{0 k}^{g n}-1 \\ \bar{v}_{k r}^{g, n-1} & \text { otherwise. }\end{cases}
$$

Now we would be done except that the updated estimate might not be convex and a procedure to restore convexity is needed. We follow the SPAR algorithm of Powell et al. (2004) for concave functions and apply it to our convex case. This method restores convexity by projecting the updated estimate of the slopes onto the space of monotone increasing functions. The projection operation is an optimization problem of the form

$$
\min _{\bar{v}_{k}^{g n}}\left\|\bar{v}_{k}^{g n}-u_{k}^{n}\right\|^{2}
$$

subject to:

$$
\bar{v}_{k, r+1}^{g n}-\bar{v}_{k r}^{g n} \geq 0
$$

which is easily solved since it involves simply averaging slopes around $R_{0 k}^{g n}$.

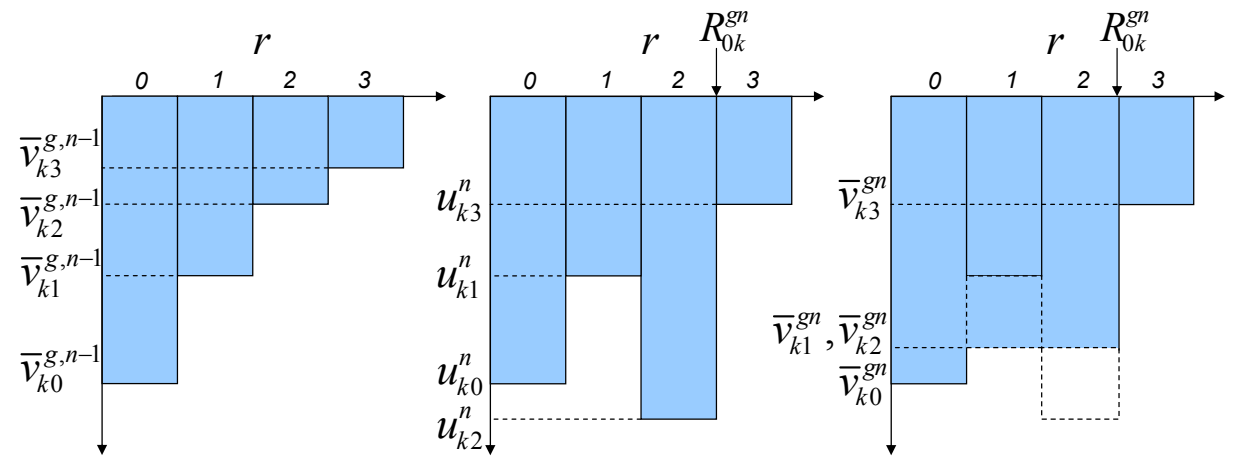

Figure 6: Illustration of SPAR with a convexity violation to the left of $R_{0 k}^{g n}=3$. The original VFA is on the left, the intermediate update in the middle, and the result of the projection on the right. The functions represent the slopes of the piecewise linear VFA term of group $k$. 
Step 0 Initialize $\bar{V}_{0}^{0}$ and set $n=1$.

Step 1 Solve 47 - 50 . If $n=N+1$ stop. $x_{0}^{N+1}$ is the solution.

Step 2a Sample $\hat{W}_{1}\left(\omega^{n}\right)$ and determine gradients:

$$
\begin{aligned}
\hat{v}^{\text {right }, n} & =\left(\hat{v}_{a}^{\text {right }, n}\right)_{a \in \mathcal{A}, R_{0 a}^{n}=0} \\
\hat{v}^{\text {left }, n} & =\left(\hat{v}_{a}^{\text {left }, n}\right)_{a \in \mathcal{A}, R_{0 a}^{n}=1}
\end{aligned}
$$

as defined in (21) and (22)

Step 2b Determine:

$$
\begin{aligned}
\hat{v}^{g, \text { right }, n} & =\left(\hat{v}_{k}^{g, \text { right }, n}\right)_{k=1,2, . ., K, R_{0 k}^{g n}<\left|\mathcal{A}_{k}\right|} \\
\hat{v}^{g, l e f t, n} & =\left(\hat{v}_{k}^{g, l e f t, n}\right)_{k=1,2, \ldots, K, R_{0 k}^{g n}>0} \\
\hat{\delta}^{n} & =\left(\hat{\delta}_{a}^{n}\right)_{a \in \mathcal{A}}
\end{aligned}
$$

as defined in (51), (52), and (53) respectively.

Step 3a For every $k=1,2, \ldots, K$ calculate vector $u_{k}^{n}=\left(u_{k r}^{n}\right)_{r<\left|\mathcal{A}_{k}\right|}$ as in 55 .

Step $3 \mathbf{b}$ For every $k$ project $u_{k}^{n}$ onto the space of monotone functions to get $\bar{v}_{k}^{g n}=\left(\bar{v}_{k r}^{g n}\right)_{r<\left|\mathcal{A}_{k}\right|}$ using (56)-(57).

Step 3c Calculate $\bar{\delta}^{n}=\left(\bar{\delta}_{a}^{n}\right)_{a \in \mathcal{A}}$ as in (54).

Set $n=n+1$ and go to Step 1 .

Figure 7: ADP algorithm for the spare allocation problem with aggregation.

Figure 6 shows the steps of the SPAR algorithm for a convexity violation to the left of $R_{0 k}^{g n}=3$. Figure 7 shows the steps of the ADP algorithm with piecewise linear VFA components and aggregation.

\section{Experimental Design}

This section describes the setup of the numerical experiments that we perform to evaluate the presented ADP algorithms and to analyze the PJM system. We are interested in the solution quality produced by four different value function approximations. We study the linear approximation of section 4.1 (abbreviated LINEAR), the quadratic approximation of section 4.2 (QUAD) and the piecewise linear approximation of section 4.3 with two different aggregations. We choose to aggregate by transmission owner (PLTO) and by location (PLLO). These are the two most natural aggregations and they differ in a key point. The aggregation by transmission owner requires a mechanism to allocate spares within each group. When 
aggregating by location the allocation within a group is not important because transfer costs to and from all the banks in a location are identical.

\subsection{The p-Median Problem as Reference Solution}

How to measure solution quality is a subtle and interesting point in this research. There is a classic deterministic discrete location problem - the p-median problem - that serves very well as a standard to compare against. In fact, with some restrictions our problem reduces to a generalized p-median problem. Since p-median problems of the size encountered in this research can be easily solved using commercial optimization software we are able to find the optimal solution for these simplified problem instances. In these cases the goal for our algorithms is to come close to optimal. In the cases where a problem instance violates pmedian assumptions we would expect to outperform the reference solution in some systematic way or at least in most cases.

We refer the reader to Mirchandani (1990) and Labbé et al. (1995) for a detailed presentation of the p-median problem. It aims to optimally locate $p$ facilities among a discrete set of possible locations. The facilities are used to satisfy demand in a discrete set of demand locations. The objective is to minimize the total sum of transportation costs between facilities and demand locations. At first sight the p-median problem looks very similar to the spare transformer problem. A closer look reveals the differences.

The facilities in the p-median problem are assumed to have infinite capacity and therefore demand is always satisfied and it is always satisfied from the "closest" facility (i.e. the one with the lowest transportation costs).

This is clearly not the case in our problem. If there is a failure and the closest spare is otherwise in use our model attempts to get the next closest spare. If there are more failures than spares then failures are left unmet. If there is a spare available to meet a failure but the transfer is not economical the failure is also left unmet. These are the three most obvious differences to the p-median problem.

We can convert our problem to a p-median problem by assuming there is at most one 
failure and the congestion costs at a failure location always outweigh the transfer costs of a spare to that location. Making these assumptions puts us in the position to obtain optimal solutions for interesting instances of our problem.

\subsection{Test Data Sets}

Table 1 describes all the data sets used in the experiments. The data sets are meant to comprise an interesting mix of data characteristics. Data sets with the prefix MU include all 71 of PJM's transformer banks in 42 substation locations. That means there are locations with multiple banks. Data sets with the prefix SI include 42 banks in 42 locations. All locations have a single bank.

Data sets MU1, MU2, SI1, SI2, and SI3 are used for testing the solution quality. The others are used in the study of section 6.3. Among the five data sets used for testing MU1, SI1 and SI2 assume a single transformer failure and our model is in this case equivalent to the corresponding p-median problem. Data sets MU2 and SI3 allow for multiple independent failures and we would expect to outperform the reference solutions in these cases. In all five data sets we use hazard rate functions estimated by PJM (Chen \& Egan (2006)) to determine transformer failure probabilities. The failure probability of a transformer depends on its age and its maintenance status which can be "good", "average", or "watch". We obtain bank failure probabilities from individual transformer failure probabilities by calculating the probability of the event that "at least one transformer in the bank fails" over the period of one year.

For the experiments, the inventory holding cost is 0 . We also assume a single transformer manufacturer and a fixed transformer purchase price of $\$ 5$ million. This leaves the parameter

$c_{1 a d}$ for $d \in \mathcal{D}^{\text {move }}$ to be considered. The corresponding decision $x_{1 a d}$ for $d \in \mathcal{D}^{\text {move }}$ implies that a spare is moved to a failure site and used to cover a failure. Thus, the cost parameter for that decision is the transfer cost minus the one-year congestion cost. The former is incurred due to the movement of the spare. The latter represents the benefit of avoided system congestion. 


\begin{tabular}{|c|c|c|c|c|c|c|c|c|c|}
\hline Name & $\begin{array}{c}\text { No. } \\
\text { Banks }\end{array}$ & $\begin{array}{l}\text { No. } \\
\text { Loc. }\end{array}$ & $\begin{array}{l}\text { Shared } \\
\text { Spares }\end{array}$ & $\begin{array}{l}\text { Failure } \\
\text { Gener. }\end{array}$ & $\begin{array}{c}\text { Exp. } \\
\text { No. } \\
\text { Failures } \\
\end{array}$ & $\begin{array}{c}\text { Transp. } \\
\text { Cost } \\
\text { (millions) } \\
\end{array}$ & $\begin{array}{c}\text { Transfer } \\
\text { Time } \\
\text { (years) } \\
\end{array}$ & $\begin{array}{l}\text { Cong. } \\
\text { Cost } \\
\text { Model } \\
\end{array}$ & $\begin{array}{c}\text { Cong. } \\
\text { Cost } \\
\left(10^{7}\right) \\
\end{array}$ \\
\hline MU1 ${ }^{*}$ & 71 & 42 & Yes & Single & 1 & n.a. & $\begin{array}{ll}\text { min: } & 0.16 \\
\text { med: } & 0.26 \\
\text { max: } & 0.43\end{array}$ & n.a. & med: 22.24 \\
\hline MU2 & 71 & 42 & Yes & Indep. & 5.2 & n.a. & $\begin{array}{ll}\min : & 0.01 \\
\text { med: } & 0.24 \\
\text { max: } & 0.63\end{array}$ & $\begin{array}{l}\text { Uniform } \\
\text { between } 12 \\
\text { and } 32\end{array}$ & med: 24.49 \\
\hline MU3 & 71 & 42 & Yes & Indep. & 2.7 & $\begin{array}{l}\text { min: } 0.23 \\
\text { med: } 1.69 \\
\text { max: } 3.45\end{array}$ & $\begin{array}{ll}\text { min: } & 0.16 \\
\text { med: } & 0.26 \\
\text { max: } & 0.43\end{array}$ & n.a. & med: 22.24 \\
\hline MU4 & 71 & 42 & No & Indep. & 2.7 & $\begin{array}{l}\text { min: } 0.23 \\
\text { med: } 1.03 \\
\text { max: } 2.15\end{array}$ & $\begin{array}{ll}\text { min: } & 0.16 \\
\text { med: } & 0.26 \\
\text { max: } & 0.43\end{array}$ & n.a. & med: 22.24 \\
\hline MU5 & 71 & 42 & Yes & Indep. & 4.1 & $\begin{array}{l}\text { min: } 0.23 \\
\text { med: } 1.69 \\
\text { max: } 3.45\end{array}$ & $\begin{array}{ll}\text { min: } & 0.16 \\
\text { med: } & 0.26 \\
\text { max: } & 0.43\end{array}$ & n.a. & med: 33.36 \\
\hline MU6 & 71 & 42 & Yes & Indep. & 2.7 & $\begin{array}{l}\text { min: } 0.23 \\
\text { med: } 1.69 \\
\text { max: } 3.45\end{array}$ & $\begin{array}{ll}\min : & 0.09 \\
\text { med: } & 0.19 \\
\text { max: } & 0.36\end{array}$ & n.a. & med: 22.24 \\
\hline $\mathrm{SI}^{*}$ & 42 & 42 & Yes & Single & 1 & n.a. & $\begin{array}{ll}\text { min: } & 0.01 \\
\text { med: } & 0.37 \\
\text { max: } & 0.98\end{array}$ & n.a. & med: 22.1 \\
\hline $\mathrm{SI} 2^{*}$ & 42 & 42 & Yes & Single & 1 & n.a. & $\begin{array}{ll}\text { min: } & 0.25 \\
\text { med: } & 0.25 \\
\text { max: } & 0.25\end{array}$ & n.a. & med: 22.1 \\
\hline SI3 & 42 & 42 & Yes & Indep. & 2.6 & n.a. & $\begin{array}{ll}\text { min: } & 0.01 \\
\text { med: } & 0.24 \\
\text { max: } & 0.63\end{array}$ & $\begin{array}{l}\text { Uniform } \\
\text { between } 12 \\
\text { and } 32\end{array}$ & med: 23.76 \\
\hline
\end{tabular}

Table 1: Data sets used for solution quality assessment.

* Equivalent to the corresponding p-median problem.

We use two different scenarios for the congestion cost. One scenario consists of congestion costs provided by PJM. In these cases we do not need a congestion cost model (denoted by n.a. in Table 1). Of course we wish to show that our methods work for more than one congestion cost scenario. The second scenario uses randomly generated congestion costs. The median congestion cost of the two scenarios are very close. But otherwise the two scenarios are very different. The real data has high variance and is skewed with a number of outliers that have very high congestion cost. The randomly generated data - coming from a uniform distribution - is much smoother.

The transfer cost contains two components, a) a transportation cost which is the cost to effect the physical movement of the spare and b) a congestion cost component that accounts for the system congestion while the spare is being transferred. The transfer time represents 
the time it takes to organize and execute the spare movement. During the transfer time the failure that initiated the spare movement is left unmet. If the transfer time is, for example, three months then the transfer cost would include one quarter of the one-year congestion cost at the destination location.

In reality little more than anecdotal evidence on actual transfer times is available. This is why we need a model to generate artificial transfer times. We use different models. Sometimes transfer times are multiples of the distance between locations, sometimes they are fixed, and sometimes they have a fixed component and a linear variable component in the distance. The location of the spares becomes more important as the variability in the transfer times increases. Thus the purely variable case makes for good test cases for our algorithms.

The data sets that are used for the solution quality assessment are set up such that a failure is always met with a spare if a spare is available. In this case the avoided congestion cost is constant for a given sample realization and number of spares. It is therefore legitimate to remove the avoided congestion costs from the objective function when evaluating two competing solutions. By evaluating only the transfer cost we can get a sharper picture of the relative solution quality than by looking at the entire objective function that includes large constant avoided congestion cost terms.

\subsection{Algorithm Tuning}

ADP algorithms would not work well without parameters that ensure a good learning behavior. The step sizes, $\alpha_{n-1}$, and the number of training iterations, $N$, are the most important parameters.

Figure 8 shows how the average solution quality changes with the number of training iterations for different problem instances using the PLLO algorithm. To obtain the graph we ran the algorithm for each instance and periodically evaluated the solution of the approximate problem. The evaluation of a solution is performed in slightly different ways. If the sample space is easily enumerable (as in our single failure experiments) then the evaluation uses all the scenarios and their probabilities to calculate the expectation of the relevant second 


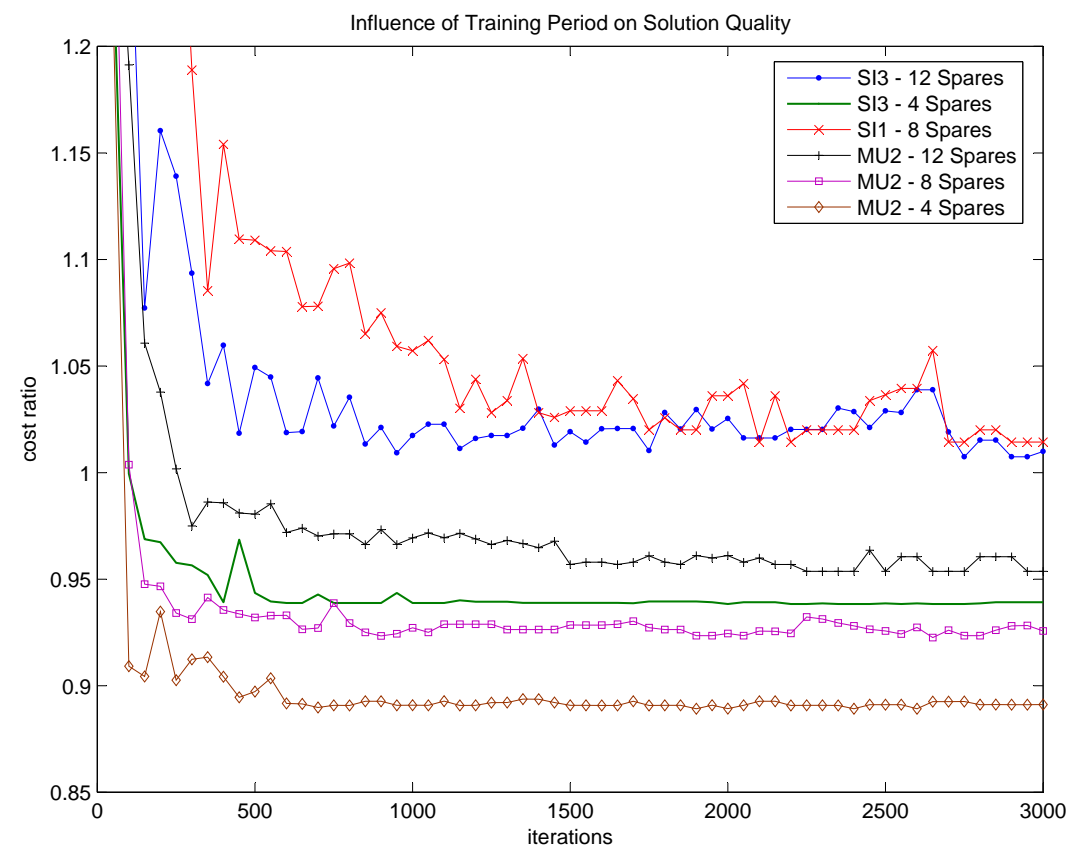

Figure 8: Average solution quality as a function of the number of training iterations for different problem instances using the PLLO algorithm. Each line is an average of 5 different sample paths where each sample path $\left(\omega^{1}, \omega^{2}, \ldots, \omega^{3000}\right)$ contains one sample for each iteration. The expected transfer cost is evaluated in increments of 50 iterations and divided by the expected transfer cost of the corresponding p-median solution to obtain the cost ratio.

stage costs. If the sample space is not enumerable we use 3000 randomly drawn, and equally weighted failure scenarios to approximate the expectation. The graphs in figure 8 show the cost ratio which is the quality of the PLLO solution divided by the quality of the corresponding p-median solution.

We see that the convergence behavior varies considerably across instances. For data set SI3 with four spares (SI3 - 4 Spares), for example, the solution quality improves and stabilizes within about 500 iterations. SI1 - 8 Spares on the other hand has much slower improvement and the solution quality also varies considerably until around iteration 2700. Given the variation in convergence behavior it is appropriate to customize the number of training iterations to each problem instance. In order to do so we run PLLO for each problem instance for multiples of 500 iterations and pick $N$ for which the average solution quality is best. Tables 3 in section 6.1 and 5 in section 6.2 show the results of this analysis. 


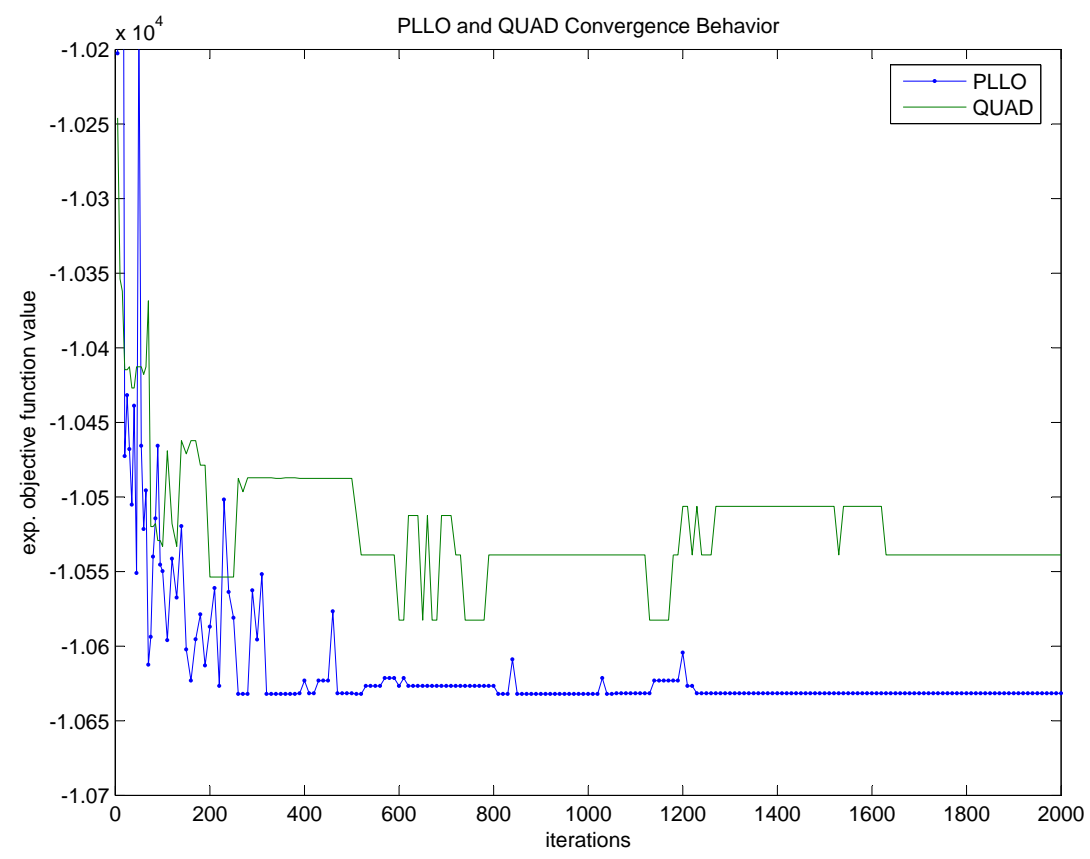

Figure 9: An example of the convergence behavior of QUAD and PLLO (data set MU3, 10 spares).

Run time considerations prevent us from using more than 600 iterations for QUAD which is adequate according to our empirical tests.

Since convergence is key in ADP algorithms we have chosen the step size rules with great care based on extensive experimental testing. For QUAD we use the quickly decreasing step sizes $\alpha_{n-1}=\frac{1}{n}$ and for LINEAR, PLTO, and PLLO we choose $\alpha_{n-1}=\frac{5}{4+n}$ which produces step sizes that decline more slowly.

As figure 8 suggests ADP algorithms do not continuously improve over the training period. For PLLO this characteristic is manageable and obtaining a very good solution is typical. This is not the case for QUAD. Figure 9 shows by example that PLLO converges more uniformly than QUAD. In the example QUAD finds a reasonable solution right around iteration 600 but does not systematically converge to it. This issue needs to be addressed in order to ensure satisfactory solution quality. We employ the simplest possible fix: QUAD periodically evaluates the solution of the approximate problem and remembers the best solution over the course of the algorithm. 
QUAD can suffer from excessive run times if the first stage MIP happens to be a difficult problem instance. To address this we cannot rely on MIP warm starts as they are not nearly as effective as LP warm starts. Instead we set a time limit of 17 seconds for the first stage MIP. Every 50 iterations we increase the limit to 60 seconds for one iteration to increase the chances of getting an optimal solution. Fortunately, the run time limit on the first stage MIP does not appear to lead to a deterioration of the solution quality.

\section{$6 \quad$ Numerical Results}

This section presents the results of three different types of experiments. In section 6.1 we carefully study the solution quality of different VFAs for data sets where the p-median solution is optimal (MU1, SI1, SI2). The goal is to show that our algorithms provide consistently near-optimal solutions. Section 6.2 contains results for more realistic data sets where the pmedian solution is not necessarily optimal (MU2, SI3). We wish to show that our algorithms outperforms the reference solution when it is not optimal. Section 6.3 investigates spare transformer allocation issues of practical interest using data sets MU3 - MU6.

\subsection{Solution Quality when the Optimal Solution Is Known}

Table 2 shows the experimental results for problem instances where p-median is optimal. We ran each algorithm for each data set varying the number of transformer spares. The solutions are evaluated as described in section 5.3, $r$ is the cost ratio which is defined as

$$
r=\frac{\text { expected transfer cost of ADP solution }}{\text { expected transfer cost of p-median solution }}
$$

$N$ gives the number of training iterations and $s$ the average run time in seconds. The entries in the $r$ and $s$ columns are averages over five different sample paths $\left(\omega^{1}, \omega^{2}, \ldots, \omega^{N}\right)$.

LINEAR works very well in cases with one bank per location. Its bad performance for cases with multiple banks reflects the bunching issue analyzed in section 4.1. PLTO does address the bunching problem to a degree but shows sometimes poor performance when the number of spares is large. 


\begin{tabular}{|c|c|ccc|ccc|ccc|ccc|}
\hline \multirow{2}{*}{$\begin{array}{c}\text { Data } \\
\text { Set }\end{array}$} & $\begin{array}{c}\text { No. } \\
\text { Spares }\end{array}$ & $r$ & $N$ & $s$ & $r$ & $N$ & $s$ & $r$ & $N$ & $s$ & $r$ & $N$ & $s$ \\
\hline \hline MU1 & 1 & 1.00 & 500 & 271 & 1.00 & 500 & 168 & 1.00 & 100 & 1041 & 1.00 & 500 & 168 \\
& 4 & 1.30 & 2000 & 646 & 1.05 & 2000 & 632 & 1.02 & 400 & 4195 & 1.00 & 1500 & 486 \\
& 8 & 1.76 & 2000 & 645 & 1.15 & 2000 & 634 & 1.04 & 400 & 4473 & 1.00 & 2000 & 651 \\
\hline SI1 & 1 & 1.00 & 500 & 42 & 1.00 & 500 & 41 & 1.00 & 100 & 166 & 1.00 & 500 & 42 \\
& 4 & 1.02 & 2000 & 161 & 1.07 & 2000 & 158 & 1.08 & 600 & 1121 & 1.02 & 1000 & 82 \\
& 8 & 1.04 & 2000 & 159 & 1.42 & 2000 & 159 & 1.14 & 600 & 2055 & 1.01 & 3000 & 242 \\
\hline SI2 & 1 & 1.01 & 500 & 40 & 1.01 & 500 & 158 & 1.01 & 100 & 161 & 1.01 & 500 & 41 \\
& 4 & 1.01 & 2000 & 161 & 1.04 & 2000 & 158 & 1.01 & 400 & 654 & 1.00 & 1500 & 121 \\
& 8 & 1.02 & 2000 & 160 & 1.16 & 2000 & 158 & 1.02 & 400 & 651 & 1.00 & 3000 & 241 \\
\hline
\end{tabular}

Table 2: Solution quality and computational effort for instances where p-median is optimal. $r$ is the ratio of the transfer cost of the ADP solution to the transfer cost of the p-median solution, $N$ is the number of iterations, and $s$ is the elapsed time in seconds. $r$ and $s$ are averages with sample size 5. Runs are measured on a single processor (Intel P4), $3.06 \mathrm{GHz}$ machine.

QUAD on the other hand produces generally good solutions even though the run times for the larger instances (MU1) are fairly high. PLLO provides excellent solutions and shows good run-time behavior. The cost ratio is always within $2 \%$ of the optimal and the largest instance solves in less than 11minutes.

\begin{tabular}{|c|c|c|c|c|c|c|c|c|}
\hline $\begin{array}{c}\text { Data } \\
\text { Set }\end{array}$ & $\begin{array}{c}\text { No. } \\
\text { Spares }\end{array}$ & 500 & 1000 & 1500 & 2000 & 2500 & 3000 & R/500it \\
\hline \hline MU1 & 1 & 1.00 & 1.00 & 1.00 & 1.00 & 1.00 & 1.00 & 166 \\
& 4 & 1.03 & 1.01 & 1.00 & 1.00 & 1.00 & 1.01 & 164 \\
& 8 & 1.06 & 1.04 & 1.03 & 1.00 & 1.01 & 1.01 & 161 \\
\hline SI1 & 1 & 1.00 & 1.00 & 1.00 & 1.00 & 1.00 & 1.00 & 41 \\
& 4 & 1.04 & 1.02 & 1.03 & 1.02 & 1.02 & 1.02 & 40 \\
& 8 & 1.09 & 1.06 & 1.03 & 1.04 & 1.04 & 1.01 & 40 \\
\hline SI2 & 1 & 1.01 & 1.01 & 1.01 & 1.01 & 1.01 & 1.01 & 40 \\
& 4 & 1.02 & 1.02 & 1.00 & 1.01 & 1.00 & 1.01 & 40 \\
& 8 & 1.07 & 1.04 & 1.02 & 1.02 & 1.02 & 1.00 & 40 \\
\hline
\end{tabular}

Table 3: Average solution quality (cost ratio) of PLLO for multiples of 500 iterations and computational effort in seconds per 500 iterations.

Table 3 quantifies the trade off between run time and solution quality for PLLO. We also use these results to pick the best number of training iterations for PLLO as described in 
section 5.3. This table shows the run times per 500 iterations. Technically, this is an average run time but for each problem instance the run times per 500 iterations is nearly constant. We see that 500 iterations are always enough to bring the solution quality within 10 percentage points of the best achievable value. The rest of the iterations is spent closing this gap.

\subsection{Solution Quality when the Optimal Solution Is Unknown}

Table 4 shows the experimental results for problem instances where p-median is not necessarily optimal.

\begin{tabular}{|c|c|ccc|ccc|cccc|ccc|}
\hline \multirow{2}{*}{$\begin{array}{c}\text { Data } \\
\text { Set }\end{array}$} & No. & \multicolumn{3}{|c|}{ LINEAR } & \multicolumn{3}{c|}{ PLTO } & \multicolumn{3}{c|}{ QUAD } & \multicolumn{3}{c|}{ PLLO } \\
\hline \hline MU2 & 1 & 0.96 & 500 & 173 & 0.96 & 500 & 171 & 0.96 & 100 & 1283 & 0.96 & 500 & 203 \\
& 4 & 0.91 & 2000 & 686 & 0.90 & 2000 & 669 & 0.89 & 400 & 13322 & 0.89 & 1000 & 371 \\
& 8 & 1.11 & 2000 & 661 & 0.94 & 2000 & 663 & 0.98 & 400 & 12690 & 0.93 & 2000 & 688 \\
& 12 & 1.50 & 2000 & 664 & 1.10 & 2000 & 653 & 1.04 & 600 & 19571 & 0.96 & 2500 & 854 \\
\hline SI3 & 1 & 1.00 & 500 & 43 & 1.00 & 500 & 42 & 1.00 & 100 & 287 & 0.99 & 500 & 60 \\
& 4 & 0.94 & 2000 & 167 & 0.98 & 2000 & 161 & 0.99 & 400 & 1872 & 0.94 & 500 & 60 \\
& 8 & 0.92 & 2000 & 168 & 1.11 & 2000 & 161 & 1.01 & 400 & 1781 & 0.92 & 2000 & 179 \\
& 12 & 1.03 & 2000 & 164 & 1.51 & 2000 & 160 & 1.10 & 400 & 2699 & 1.01 & 3000 & 260 \\
\hline
\end{tabular}

Table 4: Solution quality and computational effort for instances where p-median is not optimal. $r$ is the cost ratio, $N$ is the number of iterations, and $s$ is the elapsed time in seconds. $r$ and $s$ are averages with sample size 5 .

This table documents the problem with QUAD which are the excessive run times for most runs with data set MU2. PLLO is again the best algorithm, outperforming the p-median solution almost always and by as much as 11\%. PLLO solves instance SI3 - 12 Spares in less than 5 minutes and MU2 - 12 Spares in less than 15 minutes which indicates that the algorithm could also handle problems with more locations.

Table 5 shows the results of the convergence analysis. We see that 500 iterations are enough to bring the solution quality within 4 percentage points of the best achievable value. 


\begin{tabular}{|c|c|c|c|c|c|c|c|c|}
\hline \multirow{2}{*}{$\begin{array}{c}\text { Data } \\
\text { Set }\end{array}$} & $\begin{array}{c}\text { No. } \\
\text { Spares }\end{array}$ & 500 & 1000 & 1500 & 2000 & 2500 & 3000 & Run Time \\
\hline \hline MU2 & 1 & 0.96 & 0.96 & 0.96 & 0.96 & 0.96 & 0.96 & 172 \\
& 4 & 0.90 & 0.89 & 0.89 & 0.89 & 0.89 & 0.89 & 169 \\
& 8 & 0.94 & 0.93 & 0.93 & 0.92 & 0.93 & 0.93 & 165 \\
& 12 & 0.99 & 0.97 & 0.96 & 0.96 & 0.95 & 0.95 & 162 \\
\hline SI3 & 1 & 0.99 & 1.00 & 1.00 & 1.00 & 1.00 & 1.00 & 43 \\
& 4 & 0.94 & 0.94 & 0.94 & 0.94 & 0.94 & 0.94 & 42 \\
& 8 & 0.98 & 0.93 & 0.93 & 0.92 & 0.92 & 0.92 & 41 \\
& 12 & 1.05 & 1.02 & 1.02 & 1.03 & 1.03 & 1.01 & 40 \\
\hline
\end{tabular}

Table 5: Average solution quality (cost ratio) of PLLO for multiples of 500 iterations and computational effort in seconds per 500 iterations.

\subsection{Practical Spare Allocation Issues}

This section provides insights into four spare transformer allocation issues that are of interest to transmission owners and operators. How many spares to have in order to achieve a balance between failure risk and capital expenditures is a primary concern. We address this question by running our model repeatedly increasing the number of spares and evaluating the solutions. The differences in expected costs are the expected marginal values of spares.

Data sets MU3 - MU6 are used for this analysis. They lay claim to being a realistic in terms of failure probabilities and cost parameters - for PJM's system. In data set MU3, spares can be used without restrictions throughout the network. This assumption does not always hold in practice. For example, sharing spares among the TOs that own them is not standard practice. Spares can also have technical characteristics that make them unusable in certain network locations. With data set MU4 we analyze the situation where spares may not be shared among TOs. Once a spare is allocated to a TO it can only be used to address failures in this TO's territory. In all other respects MU4 is identical to MU3.

Ordering lead times for new transformers are also a concern in the industry. Production capacity is limited. High demand, labor strikes, copper shortages, and other uncertain events can push ordering lead times beyond 18 or even 24 months. In MU3 the failure probabilities and the avoided congestion costs are calculated for a 12 month horizon which can be 


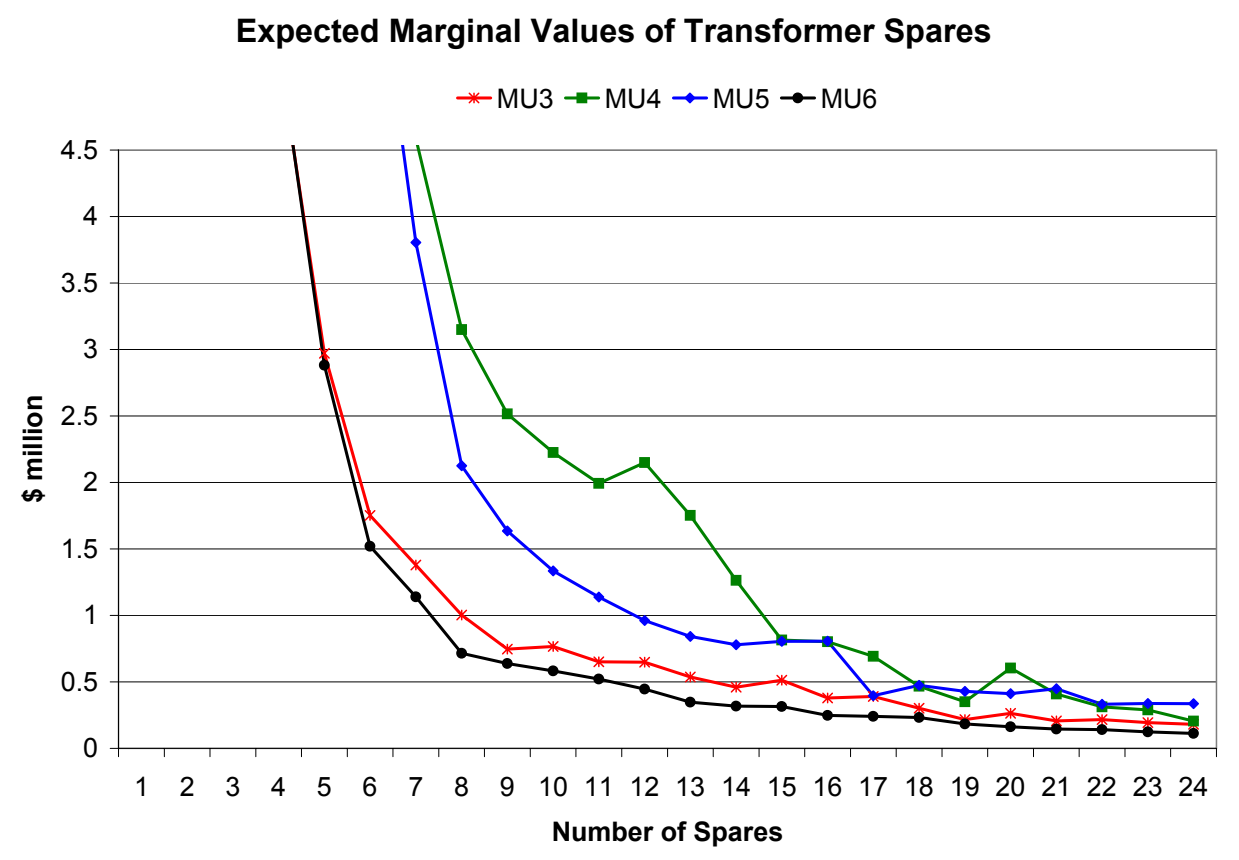

Figure 10: Expected marginal values of transformer spares as a function of the number of spares. Each data point is an average over five sample paths $\left(\omega^{1}, \omega^{1}, \ldots, \omega^{N}\right)$ where $N=2000$.

interpreted as a 12 month ordering lead time. MU5 uses an 18 month horizon instead.

Finally, it is of interest to explore the effect of switchable spares. Any spare can be preprepared for service at the substation where it is stored. Turning on such a switchable spare is a matter of a few days. If a spare is not switchable positioning within the substation and putting it in service can take up to one month. Data set MU3 assumes switchable spares that take 5 days to put into service. MU6 assumes non-switchable spares which require 30 days of preparation. It is important to stress that this distinction is only relevant for substations with an on-site spare.

Figure 10 shows the results of the marginal value analysis. In order to determine the optimal number of spares one looks for the point where the marginal value hits the marginal cost of a spare. The cost of a spare has to be prorated to match the one year time horizon of the model.

As would be expected, the sharing of spares (MU3 vs. MU4) has a strong impact on the 
optimal number. Assuming a marginal cost of $\$ 1$ million the optimal number is 8 with sharing and between 14 and 15 without. If the marginal cost is $\$ 500,000$ the numbers are 14 and 18 respectively. The relative effect of transformer sharing decreases as the marginal cost go down.

Comparing MU3 with MU5 we see that the effect of the ordering lead time on the optimal number is relatively small and also shrinks as the marginal cost decreases. If the marginal cost is $\$ 1$ million $/ \$ 1.5$ million for MU3/MU5 the optimal number is 8 for MU3 and between 9 and 10 for MU5. If the marginal cost is $\$ 500,000 / \$ 750,000$ then the optimal number is approximately 14 for MU3 and 15 for MU5.

The curve for MU3 dominates the curve for MU6. That means that if spares are switchable the optimal number increases. The intuitive interpretation of this behavior is that with switchable spares it is very desirable to have an on-site spare as opposed to bringing in an off-

site spare. With additional spares the model can potentially grab more of the on-site/off-site transfer cost difference. Since this difference is big if spares are switchable it can justify more spares.

\section{Conclusions and Further Research}

In this paper we have introduced a two-stage spare transformer allocation model. Current technology is far from being able to solve large realistic instances of this problem exactly. We use an ADP approach to solve the model approximately and observe that VFAs are needed that can take into account the non-separable behavior of the true value function. We introduce two such VFAs and test the obtainable solution quality. PLLO is the best algorithm. It consistently produces very good solutions and solves very efficiently. We show that the model can be used to answer spare transformer allocation questions of practical interest.

A useful extension of this research is the introduction of multi-period models that reflect the true dynamic nature of high-voltage transformer management. They could be used to model the transformer population and the electric grid as they change over time and answer questions about the timing of transformer purchases and replacements, spare deployment 
policies, and many others. The performance characteristics of the algorithm used in this paper are so good that it would be a suitable algorithmic starting point for the richer and larger multi-period transformer management models. 


\section{References}

Bertsekas, D. \& Tsitsiklis, J. (1996), Neuro-Dynamic Programming, Athena Scientific, Belmont, MA. 2

Birge, J. \& Louveaux, F. (1997), Introduction to Stochastic Programming, Springer-Verlag, New York. 2

Chen, Q. M. \& Egan, D. M. (2006), 'A bayesian method for transformer life estimation using perks' hazard function', IEEE Transactions on Power Systems 21(4), 1954-1965. 25

Chowdhury, A. A. \& Koval, D. O. (2005), 'Development of probabilistic models for computing optimal distribution substation spare transformers', IEEE Transactions on Industry Applications 41(6), 1493-1498. 3

Godfrey, G. \& Powell, W. B. (2002), 'An adaptive, dynamic programming algorithm for stochastic resource allocation problems I: Single period travel times', Transportation Science 36(1), 21-39. 3. 19

Godfrey, G. A. \& Powell, W. B. (2001), 'An adaptive, distribution-free approximation for the newsvendor problem with censored demands, with applications to inventory and distribution problems', Management Science 47(8), 1101-1112. 19, 21.

Helmberg, C. (2000), Semidefinite programming for combinatorial optimization, Technical report, Konrad-Zuse-Zentrum fuer Informationstechnik Berlin, Berlin. 15

Kall, P. \& Wallace, S. (1994), Stochastic Programming, John Wiley \& Sons, New York. 2

Kogan, V. I., Roeger, C. J. \& Tipton, D. E. (1996), 'Substation distribution transformers failures and spares', IEEE Transactions on Power Systems 11(4), 1905-1912. 3

Labbé, M., Peeters, D. \& Thisse, J.-F. (1995), Location on networks, in M. Ball, T. L. Magnanti, C. L. Monma \& G. L. Nemhauser, eds, 'Handbooks in Operations research and Management Science: Network Routing', Elsevier, Amsterdam. 3, 24

Laporte, G., Louveaux, F. V. \& van Hamme, L. (1994), 'Excact solution to a location problem with stochastic demands', Transportation Science 28(2), 95-103. 2, 7

Li, W., Vaahedi, E. \& Mansour, Y. (1999), 'Determining number and timing of substation spare transformers using a probabilistic cost analysis approach', IEEE Transactions on Power Delivery 14(3), 934-939. 3

Louveaux, F. V. \& Peeters, D. (1992), 'A dual-based procedure for stochastic facility location', Operations Research 40(3), 564-573. 2, 7

Mirchandani, P. B. (1990), The p-median problem and generalizations, in P. B. Mirchandani \& R. L. Francis, eds, 'Discrete Location Theory', Wiley, New York. 3, 24

Ntaimo, L. \& Sen, S. (2005), "The million-variable "march" for stochastic combinatorial optimization', Journal of Global Optimization 32, 385-400. 2, 3, 7

Powell, W. B. (2007), Approximate Dynamic Programming: Solving the curses of dimensionality, John Wiley and Sons, New York. 8 
Powell, W. B. \& Topaloglu, H. (2004), Fleet management, in S. Wallace \& W. Ziemba, eds, 'Applications of Stochastic Programming', Math Programming Society - SIAM Series in Optimization, Philadelphia. 3

Powell, W. B. \& Van Roy, B. (2004), Approximate dynamic programming for high dimensional resource allocation problems, in J. Si, A. G. Barto, W. B. Powell \& D. W. II, eds, 'Handbook of Learning and Approximate Dynamic Programming', IEEE Press, New York. 8

Powell, W. B., George, A., Bouzaiene-Ayari, B. \& Simao, H. (2005), Approximate dynamic programming for high dimensional resource allocation problems, in 'Proceedings of the IJCNN', IEEE Press, New York. 2

Powell, W. B., Ruszczyński, A. \& Topaloglu, H. (2004), 'Learning algorithms for separable approximations of stochastic optimization problems', Mathematics of Operations Research 29(4), 814-836. 3, 21, 22

Powell, W. B., Shapiro, J. A. \& Simão, H. P. (2002), 'An adaptive dynamic programming algorithm for the heterogeneous resource allocation problem', Transportation Science 36(2), 231-249. 8, 11

Puterman, M. L. (1994), Markov Decision Processes, John Wiley \& Sons, New York. 8

Sen, S. (2005), Algorithms for stochastic mixed-integer programming models, in K. Aardal, G. L. Nemhauser \& R. Weismantel, eds, 'Handbooks in Operations Research and Management Science: Discrete Optimization', North Holland, Amsterdam. 2

Topaloglu, H. (2001), Dynamic programming approximations for dynamic programming problems, Ph.d. dissertation, Department of Operations Research and Financial Engineering, Princeton University. 3

Topaloglu, H. \& Powell, W. B. (2003), 'An algorithm for approximating piecewise linear concave functions from sample gradients', Operations Research Letters 31(1), 66-76. 21

Topaloglu, H. \& Powell, W. B. (2006), 'Dynamic programming approximations for stochastic, time-staged integer multicommodity flow problems', Informs Journal on Computing 18(1), 31-42. 2, 3, 8 